\title{
Los movimientos oculares como herramienta metodológica para el estudio de las dificultades de lectura en niños con TDAH ${ }^{1}$
}

\section{Os movimentos oculares como ferramenta metodológica para o estudo} das dificuldades de leitura em crianças com TDAH

Eye tracking as a methodology for the study of reading difficulties in ADHD children

Inmaculada Escudero², Paloma Martínez ${ }^{3}$, José Antonio León ${ }^{4}$

Este trabajo ha sido financiado parcialmente por los proyectos MINNECO PSI2013 47219-P (del Ministerio de Economía Competitividad)

Universidad Nacional de Educación Distancia (UNED)

E-mail: iescudero@psi.uned.es

Universidad Nacional de Educación Distancia (UNED).

E-mail: palomamartinez05@gmail.com

Eniversidad Autonoma de Madrid
RESUMEN: Este trabajo tiene como objetivo mostrar la utilidad que la metodología de movimientos oculares tiene para el estudio del TDAH y, más concretamente, para analizar las dificultades de lectura que muestran los niños con TDAH. Para ello, realizamos una breve exposición de los principales problemas de lectura detectados en esta población. Continuaremos con una exposición de los principales trabajos realizados hasta el momento, centrados en analizar los movimientos oculares en la realización de diversas tareas cognitivas en sujetos con TDAH para pasar seguidamente, al ámbito de la lectura. Finalizamos el artículo con una breve discusión sobre algunas de las implicaciones clínicas, psicológicas y educativas que conlleva el uso de esta metodología para el estudio de la lectura en esta población.

Palabras Clave: TDAH; Comprensión lectora; Movimientos oculares

RESUMO: Este trabalho objetiva mostrar a utilidade que a metodologia de movimentos oculares possui para o estudo do TDAH, mais especificamente, para analisar as dificuldades de leitura em crianças com TDAH. Para tanto, realizamos uma breve exposição dos principais problemas de leitura percebidos nessa população. Fazemos, ademais, uma apresentação das principais pesquisas realizadas até o momento, centradas em analisar os movimentos oculares na realização de diversas tarefas cognitivas em sujeitos com TDAH para, em seguida, passar ao âmbito da leitura. Na sequência, finalizamos o artigo com uma breve discussão sobre algumas das implicações clínicas, psicológicas e educativas advindas dessa metodologia no que tange ao estudo da leitura em crianças com TDAH.

Palavras-chave: TDAH; compreensão leitora; movimentos oculares.

ABSTRACT: This work aims to show the usefulness of the methodology of eye tracking for the study of ADHD and, more specifically, to analyze the reading difficulties of children with ADHD. We present a brief summary of the main reading problems detected in this population. We will continue with a discussion about research on analyzing the eye movements in performing various cognitive tasks in subjects with ADHD, focusing on the area of reading. We end the article with a brief discussion of some of the clinical, psychological and educational implications of the use of this methodology for the study of reading in this population.

KEYWORDS: ADHD; Reading comprehension; Eye tracking 


\section{Introducción}

Ll Trastorno por Déficit de Atención con Hiperactividad es uno de los trastornos con un alta prevalencia en la edad escolar, así como también lo son las dificultades de lectura. Ambas no tienen que ir necesariamente unidas pero, en muchas ocasiones, se encuentran íntimamente relacionadas. Se estima que la prevalencia del TDAH se sitúa en torno al 5\% (APA, 2013). Este trastorno se caracteriza principalmente por síntomas de inatención, hiperactividad e impulsividad, que interfieren de manera significativa con el desempeño de la persona en su vida diaria (BARKLEY, 2006). La naturaleza del TDAH a menudo causa al niño que lo sufre problemas de rendimiento escolar derivados de la inatención, la dificultad para seguir instrucciones o para organizarse (ORJALES, 2011). Hoy sabemos que, además de estas dificultades, el TDAH presenta alta comorbilidad con trastornos del aprendizaje (ATIENZA, 2006), así como dificultades de lectura. La comorbilidad entre estos aspectos y el TDAH se sitúa en torno al 25-35\% (MAYES, CALHOUN y CROWELL, 2000).

Las dificultades académicas que muestran los niños con TDAH se deben, en parte, a los síntomas de inatención e impulsividad/hiperactividad. Pero, además, estas dificultades vienen explicadas por la existencia de déficits en procesos cognitivos de orden superior (LORCH, BERTHIAUME, MILICH y VAN DEN BROEK, 2007). Una forma de aproximarse al análisis de los procesos de orden superior es, precisamente, el estudio de la comprensión narrativa. Son diversos los estudios que han mostrado que la comprensión narrativa temprana es tan importante que puede llegar a predecir el desempeño futuro en lectura (e.g., KENDEOU, BOHNGETTLER, WHITE y VAN DEN BROEK, 2008). Existe basta evidencia empírica que indica que los niños con TDAH muestran déficits específicos e importante en dicha competencia (e.g., LORCH, BERTHIAUME,
MILICH y VAN DEL BROEK, 2007; VAN NESTE, HAYDEN, LORCH y MILICH, 2015).

Dentro del estudio de la lectura, los movimientos oculares se han erigido como una metodología de gran relevancia para analizar los procesos cognitivos subyacentes. Entre sus principales ventajas cabe destacar que es una metodología de gran objetividad, ya que proporciona datos cuantitativos sobre los procesos implicados en la lectura y las habilidades visuales y atencionales (RAYNER, 1998, 2009). La investigación existente hasta el momento muestra que los movimientos oculares permiten analizar de forma precisa los procesos cognitivos que se realizan durante la lectura; esto es, suponen una importante fuente de información fiable sobre los procesos cognitivos necesarios para una buena comprensión. Entre las principales medidas utilizadas se encuentran los movimientos sacádicos, las fijaciones y las regresiones. A partir de estas medidas básicas es posible extraer todo un conjunto de medidas complementarias que aportan información importante según los objetivos de cada investigación. Así, desde el número de fijaciones y regresiones, que pueden aportar información sobre la dificultad del texto o las habilidades del lector, hasta las áreas de interés en función de dónde se fija la mirada, o los cambios de focalización, aportan datos que facilitan la comprensión de los procesos cognitivos subyacentes a la lectura.

Desde comienzos de los años 90 del siglo XX existen trabajos que han investigado las características de los movimientos oculares en el TDAH, especialmente en taras de seguimiento de estímulos visuales (visual tracking) (e.g., BYLSMA y PIVIK, 1989; ROSS, HOMMER, BREIGER, VARLEY y RADANT, 1994; ROTHILD, POSNER y SCHANGHENCY, 1991). Las diversas investigaciones realizadas, diseñadas para explorar hipótesis muy variadas, parten todas ellas de una premisa común: los movimientos oculares son un indicador observable y cuantificable de los procesos cognitivos subyacentes, supuestamente alterados en el TDAH. Los movimientos 
oculares, o sacádicos, proporcionan una medida esencial de las respuestas inhibitorias, ya que fallos en la inhibición están directamente relacionados con respuestas oculares erróneas (VAN DER STIGCHEL, ROMMELSE, DEIJEN, GELDOF, WITLOX, OOSTERLAAN, SERGEANT y THEEUWES, 2007). En este sentido, existe basta evidencia empírica que muestra que el control de los movimientos oculares está alterado en niños diagnosticados de TDAH, mostrando patrones diferentes, en comparación con otros niños sin TDAH (e.g., FEIFEL, FARBER, CLEMENTZ, PERRY y ANLLO-VENTO, 2004; GOULD, BASTAIN, ISRAEL, HOMMER y CASTELLANOS, 2001; KLEIN, RASCHKE y BRANDENBUSCH, 2003; MUNOZ, ARMSTRONG, HAMPTON y MOORE, 2003).

Pero, si bien esta metodología ha mostrado ser muy útil para analizar los déficits que muestran estos niños, apenas existen estudios sobre movimientos oculares y lectura en niños con TDAH. Quizás esto pueda deberse, en parte, al movimiento excesivo que presentan estos niños, aspecto que puede dificultar la recogida de datos, provocando una pérdida importante de información durante el registro. Sin embargo, sería importante trabajar en la forma de poder solventar o minimizar estas dificultades, ya que conocer cómo son los patrones de movimientos oculares en niños con TDAH durante la lectura nos permitiría poder ampliar nuestro conocimiento sobre la naturaleza de las dificultades que muestran estos niños $\mathrm{y}$, de forma añadida, poder intervenir, realizar las adaptaciones necesarias en los textos, desarrollar aplicaciones multimedia adaptadas...

Con el objetivo de mostrar la utilidad que esta metodología puede tener para el estudio de la lectura ante la presencia de TDAH, en este artículo realizaremos una breve exposición de los principales problemas de lectura detectados en esta población. Continuaremos con una exposición de los principales trabajos realizados hasta el momento, centrados en analizar los movimientos oculares en la realización de diversas tareas cognitivas en sujetos con TDAH para pasar, seguidamente, al ámbito de la lectura.
Finalizaremos el artículo con una breve discusión sobre algunas de las implicaciones clínicas, psicológicas y educativas que conlleva el uso de esta metodología para el estudio de la lectura en esta población.

\section{El TDAH y los problemas de lectura}

La lectura es una actividad compleja que requiere de diversas habilidades de naturaleza lingüística, cognitiva y metacognitiva (ESCUDERO, LEÓN y MORERA, 2013; LEÓN y ESCUDERO, 2015). Por tanto, comprender correctamente un texto dependerá de que las diferentes habilidades que intervienen en su procesamiento sean empleadas de manera adecuada. En este sentido, las investigaciones más recientes establecen una importante relación entre la comprensión lectora y el funcionamiento ejecutivo, que parece estar afectado en el TDAH (MIRANDA, FERNÁNDEZ, GARCÍA, ROSELLÓ y COLOMER, 2011; MIRANDA, GARCÍA y SORIANO, 2005).

En los últimos años se ha llegado a un consenso según el cual, detrás de las manifestaciones del TDAH, se encuentran alteraciones en las funciones ejecutivas cerebrales. Éstas se asientan en los lóbulos frontales del cerebro y se refieren a un conjunto de procesos cognitivos y metacognitivos que implican a la atención sostenida, la inhibición de interferencias, la planificación, el control de la conducta y la flexibilidad cognitiva (VAQUERIZO-MADRID, ESTÉVEZ-DÍAZ y POZO-GARCÍA, 2005). Por lo tanto, la afectación de la función ejecutiva se manifestará en los sujetos afectados con bajos niveles de rendimiento, problemas para concentrarse y para mantener la atención continuadamente, impulsividad y rigidez mental. Centrándose en el ámbito de la lectura, es evidente que esta disfunción ejecutiva causará problemas a los niños con TDAH ya que, para una correcta comprensión, los lectores han de hacer uso de su memoria de trabajo, sus habilidades de planificación y su capacidad para ignorar la información irrelevante y separarla 
de la que sí lo es. Dado que todas estas funciones están afectadas en el TDAH, es más probable que estos niños y niñas tengan problemas para realizar una lectura de calidad. Entre los principales problemas detectados en estos niños cabe destacar, entre otros, dificultades para juzgar el contenido de un texto, sintetizar, resumir, esquematizar, realizar inferencias, comprender las conexiones causales o las metas de los personajes, así como distinguir la información relevante (LORCH y cols., 2007; LORCH, DIENER, SÁNCHEZ, MILICH, WELSH y VAN DEL BROEK, 1999; MIRANDA y cols., 2005; RENZ, LORCH, MILICH, LEMBERGER, BODNER Y WELSH, 2003; VAN NESTE y cols., 2015).

Gracias a las investigaciones realizadas hasta el momento sabemos, además, que el déficit en las funciones ejecutivas no influye únicamente en el aprendizaje de la lectura, sino también en las competencias narrativas orales y escritas de estos alumnos (VAQUERIZO-MADRID, ESTÉVEZ-DÍAZ y POZO-GARCÍA, 2005). Es interesante analizar los déficits en las competencias narrativas de los estudiantes con TDAH, ya que éstos repercuten directamente en otros aprendizajes escolares, como la comprensión y composición de textos escritos (MIRANDA, GARCÍA y SORIANO, 2005). Además, el análisis de las competencias narrativas permite entender mejor el funcionamiento cognitivo de los niños con TDAH, ya que éstas requieren de la puesta en marcha de múltiples estrategias dependientes de las funciones ejecutivas: focalización de la atención, selección, codificación e interpretación de la información relevante, utilización de las estructuras organizativas de la historia, generación de inferencias que permitan la interpretación de la información presentada, y el uso eficaz de habilidades de recuperación (MIRANDA, GARCíA y SORIANO, 2005). En esta línea, Miranda y colaboradores diseñaron un estudio para comparar las habilidades narrativas de un grupo de estudiantes con TDAH con un grupo control igualado en edad, rendimiento escolar y CI verbal. Ambos grupos no difirieron en el número de las proposiciones que recordaron, pero sí en la calidad de las mismas, que fue superior en el grupo control. Además, los niños del grupo de TDAH presentaron mayor número de confusiones (personajes-acción) y de embellecimientos o inclusión de información no presente en el relato original. En términos generales, puede decirse que los niños con TDAH realizaron narraciones más pobres, al omitir partes esenciales de la estructura introducción-suceso-ejecución y, a su vez, tuvieron dificultades para ignorar partes irrelevantes de la historia. Estos errores avalarían la hipótesis de que las funciones ejecutivas estás afectadas en estos niños; en este caso, la memoria de trabajo y la capacidad para inhibir respuestas irrelevantes para la tarea, respectivamente.

Autores como Willcutt, Pennington, Olson y DeFries (2007) se han propuesto explorar la posible etiología común del TDAH y las dificultades en el aprendizaje. Según estos autores, el hecho de que entre el 25 y el $40 \%$ de los niños y jóvenes diagnosticados de dificultades de lectura presente también TDAH, significa que ambos trastornos pueden compartir un mismo origen. Sin embargo, Willcutt y colaboradores (2007) exponen que, una vez que los niños han iniciado la instrucción formal, es difícil dilucidar si dicha asociación comparte un origen común, o un trastorno propicia la aparición del otro. Estos autores plantean que, en algunos alumnos, la falta de atención propia del TDAH puede ocasionar que el estudiante no atienda durante las lecciones y, por tanto, no adquiera correctamente los mecanismos lectores, desarrollando un trastorno del aprendizaje de la lectura. En otros casos puede que, al no ser el niño capaz de leer correctamente, éste se distraiga en el aula y comience a exhibir comportamientos de inatención o hiperactividad. Por eso creen que la mejor manera de dilucidar esta cuestión es estudiando las habilidades prelectoras de los niños, ya que estas aún no se han visto modificadas por la instrucción formal. En el citado estudio, estos autores evaluaron los síntomas de TDAH y las habilidades prelectoras en una muestra de gemelos en edad preescolar. Las habilidades prelectoras se evaluaron a través de diferentes tareas como la identificación de rimas, el 
nombrado de colores y objetos lo más rápido posible, la identificación de letras, etc. Encontraron una pequeña pero significativa correlación entre los síntomas de inatención del TDAH y un peor desempeño en las tareas prelectoras. Sin embargo, no encontraron relación entre éstas y los síntomas de hiperactividad-impulsividad.

\section{Movimientos oculares}

\subsection{Movimientos oculares y lectura}

Como ya hemos destacado anteriormente, el estudio de los movimientos oculares durante el proceso lector aporta información muy relevante sobre los procesos implicados e, incluso, sobre las dificultades encontradas durante el mismo, a través de sus distintas medidas. Veamos brevemente cuáles son algunas de las medidas más utilizadas (véase, para una revisión más extensa, HOLMQVIST y NYSTRÖM, 2011). En primer lugar, están los movimientos sacádicos. Los movimientos sacádicos son movimientos rápidos que permiten a los ojos moverse de un punto de fijación a otro, entre los cuales se reconoce y procesa la información. Durante la lectura, se refieren a la forma en que los ojos se desplazan de izquierda a derecha. Aunque pueden variar en longitud, durante la lectura en silencio suelen abarcar de 7 a 9 caracteres, y su duración oscila entre los 20 y 40 milisegundos. Los movimientos sacádicos se van alternando con momentos de fijación, permitiendo al lector saltar de un punto a otro de forma rápida y discontinua. En función del proceso cognitivo implicado, la mirada se centra en un determinado fragmento mientras se produce el reconocimiento visual de la palabra, salta a otro fragmento diferente o, en ocasiones, se desplaza hacia atrás para releer alguna información del texto (regresiones).

En segundo lugar, están las fijaciones. Estas se refieren a los movimientos que se realizan cuando el ojo está relativamente quieto y centrado en un objetivo particular. Las fijaciones suelen durar entre 200-300 milisegundos de promedio, pudiendo llegar a oscilar entre los 100 y más de 500 milisegundos. En aquellas tareas en las que se pide al participante que lea en silencio, la duración media de una fijación es de 225 milisegundos. Suponen el $90 \%$ de tiempo de lectura y durante las mismas el lector enfoca su fóvea hacia un fragmento textual (unos siete caracteres de promedio), durante el cual se analiza la información. En este periodo, los ojos únicamente presentan unos breves temblores involuntarios, quedando prácticamente inmóviles mientras se produce el análisis visual de la palabra y los procesos cognitivos subyacentes. Además, estas fijaciones dependerán del tipo de texto (más fijaciones en función de la dificultad del mismo), del lector (lectores hábiles menos fijaciones más cortas y menos regresiones), del tipo de palabras (palabras contenido más fijaciones que las funcionales), etc.

Y, por último, las regresiones. Éstas son pequeños movimientos sacádicos hacia la izquierda que se realizan cuando una persona tiene que volver a leer una sección del texto. Suelen producirse cuando un sacádico es demasiado rápido o abarca más información que la que el sujeto puede percibir o procesar. Aproximadamente el $10-15 \%$ de todos los movimientos sacádicos son regresiones.

Gracias a revisiones importantes como la realizada recientemente por Blythe y Joseph (2011), sabemos que los movimientos oculares parecen alcanzar el nivel del adulto a la edad de 10-12 años; esto es, que en gran parte de los indicadores analizados en los estudios recogidos en dicha revisión, los niños alcanzan valores similares o cercanos a los de los adultos en torno a esta edad. Tal es el caso de la amplitud sacádica (MCCONKIE y cols., 1991), las regresiones por frase (RAYNER, 1986) o las fijaciones (TAYLOR, 1965). Blythe y Joseph señalan, asimismo, que, a medida que aumenta la edad, también mejoran las habilidades cognitivas y, por 
tanto, el progreso en la lectura. Esta mejora en las habilidades origina cambios en los patrones de movimientos oculares, reflejando éstos dicha progresión.

Son diversos los estudios que muestran la utilidad del estudio de los patrones de movimientos oculares para valorar las dificultades en el proceso lector. Así, autores como Rayner $(1998,2009)$ muestran cómo los lectores que están aprendiendo, al igual que los sujetos disléxicos, presentan fijaciones más largas y mayor número de regresiones que los lectores más hábiles. Además, apunta hacia la importancia del estímulo lingüístico, de forma que, a medida que aumenta la dificultad del texto, aumentan las regresiones y los movimientos sacádicos se hacen más cortos. También señala que, al releer, las fijaciones son más cortas y los movimientos sacádicos más largos, además de haber menos regresiones. Esto convierte el análisis de los movimientos oculares en una herramienta sumamente útil para analizar la dificultad que tiene el texto para el lector y los procesos que están implicados. Además, señala que el control de los movimientos oculares y el entrenamiento en dónde y cuándo mover los ojos, y dónde fijar la posición de la mirada, pueden favorecer una mejor lectura.

\subsection{Movimientos oculares en el TDAH}

La técnica de movimientos oculares se ha aplicado al estudio del TDAH desde principios de los años 90 del siglo XX. Son diversos los investigadores que han tratado de analizar si los niños afectados de TDAH tienen un patrón ocular característico y diferente al resto de niños sin TDAH, si bien la mayor parte se han centrado en el empleo de tareas de seguimiento visual (visual tracking). Todas estas investigaciones comparten una misma premisa: los movimientos oculares son un indicador observable y cuantificable de procesos cognitivos subyacentes y, por lo tanto, pueden utilizarse para revelar alteraciones propias de diversos trastornos. En el caso del TDAH existe basta evidencia que muestra que el control de los movimientos oculares resulta alterado en niños afectados con este trastorno, mostrando patrones diferentes, en comparación con otros niños sin TDAH (e.g., FEIFEL, FARBER, CLEMENTZ, PERRY y ANLLO-VENTO, 2004; GOULD, BASTAIN, ISRAEL, HOMMER y CASTELLANOS, 2001; KLEIN, RASCHKE Y BRANDENBUSCH, 2003; MUNOZ, ARMSTRONG, HAMPTON y MOORE, 2003). A continuación describimos brevemente los principales tipos de tareas utilizadas con la técnica de movimientos oculares con relación al TDAH:

- Tarea de fijación: Este tipo de tarea consiste en que el sujeto mantenga su vista fija en un determinado estímulo, por ejemplo, un círculo negro ubicado en el centro de la pantalla. A este estímulo se le denomina inicial y, como se expone a continuación, frecuentemente supone el punto de partida para otro tipo de tareas. En las tareas de fijación a menudo se presentan distractores para tratar de apartar la atención del sujeto del estímulo inicial. Diversas investigaciones han demostrado que los sujetos con TDAH presentan más prosacádicos intrusivos que los sujetos no afectados cuando se trata de realizar fijaciones por periodos prolongados de tiempo (e.g., MUNOZ y cols., 2003).

- Tarea prosacádica: En esta tarea se solicita a los participantes que, en primer lugar, fijen la vista en un estímulo inicial que habitualmente se sitúa en el centro de la pantalla. Posteriormente, los sujetos han de dirigir su mirada hacia donde aparezca un nuevo estímulo objetivo o el propio estímulo inicial que se ha desplazado. Los movimientos sacádicos requeridos pueden ser elicitados en horizontal, vertical o diagonal, en función de dónde se sitúe el segundo estímulo. Con este tipo de tarea se pueden medir distintos parámetros como tiempo de reacción sacádico, precisión sacádica o variabilidad inter-sujetos en 
los tiempos de reacción (HANISCH, RADACH, HOLTKAMP, HERPERTZDAHLMANN y KONRAD, 2006).

- Tarea antisacádica: Esta tarea se utiliza para comprobar la habilidad de los sujetos para suprimir movimientos sacádicos reflejos y, en su lugar, generar sacádicos voluntarios (MUNOZ y cols., 2003). A los participantes se les presenta de manera repentina un estímulo visual en la periferia del estímulo inicial. Previamente, se les habrá indicado que, cuando esto ocurra, deben dirigir su vista hacia la dirección opuesta y simétrica a la que aparece el nuevo estímulo. Esto es, si el nuevo estímulo aparece a la izquierda del estímulo inicial, el sujeto tendrá que mirar a la derecha a una distancia equivalente (BUCCI, SEASSAU, LARGER, BUI-QUOC y GERARD, 2014). La realización correcta de esta tarea requiere de dos procesos cognitivos diferentes: por un lado, la inhibición de la respuesta automática que evoca la aparición del nuevo estímulo y, por otro, la ejecución de un movimiento ocular voluntario dirigido a la localización en espejo del estímulo inicial (VAN DER STIGCHEL y cols., 2007). Las medida que típicamente se extrae de este tipo de tareas es el número de errores direccionales que comete el sujeto (ROMMELSE, VAN DER STIGCHEL y SERGEANT, 2008). Investigaciones llevadas a cabo paralelamente con técnicas de neuroimagen demuestran que, durante las tareas antisacádicas, intervienen regiones frontales del cerebro, por lo que los errores direccionales pueden considerarse un indicador de disfunciones frontales.

- Tarea de contraorden: Armstrong y Munoz (2003) utilizan esta tarea en una de sus investigaciones. La tarea incluía dos condiciones: en el 75\% de los ensayos, los sujetos debían fijar su mirada en un estímulo inicial o marcador central y, posteriormente, dirigir la vista a un estímulo periférico que aparecía a la vez que desaparecía el punto de fijación inicial. Sin embargo, en el 25\% de los ensayos, tras la aparición del estímulo periférico, una señal indicaba que los sujetos debían frenar su movimiento ocular ya iniciado y mantener la mirada sobre el marcador central de fijación. Con esta tarea los autores trataban de medir la impulsividad de los sujetos con TDAH, que hipotetizaron, tendrían menos éxito en detener sus movimientos sacádicos tras la señal de freno.

- Tarea de búsqueda visual: Este tipo de tarea se utiliza para investigar la eficiencia de la atención selectiva. Consiste básicamente en detectar un determinado estímulo (una forma concreta, cierto color, etc.) de entre una variedad de elementos distractores. El sujeto debe ser capaz de ignorar los distractores y persistir en la búsqueda del estímulo objetivo (VAN DER STIGCHEL y cols., 2007).

- Tarea de captación oculomotora (Oculomotor capture task): En la tarea de captación oculomotora se solicita a los sujetos que dirijan su mirada hacia aquel estímulo que cambie en un determinado momento (véase Figura 1). Por ejemplo, el círculo blanco, de entre varios con las mismas características, que en determinado momento pasa a ser gris. En algunos de los ensayos los participantes han de ser capaces de ignorar otro estímulo que aparece simultáneamente al estímulo objetivo, y que actúa como distractor. En el ejemplo anterior, a la vez que el círculo rojo cambia de color, aparece repentinamente un nuevo círculo en la pantalla (segunda condición de la Figura 1). La tarea recibe el nombre de captación oculomotora porque los ojos son "captados" por el estímulo distractor. Con esta tarea se trata de explorar si los sujetos con TDAH presentan mayores dificultades que el grupo control a la hora de suprimir respuestas automáticas hacia el estímulo distractor que es irrelevante para la tarea (VAN DER STIGCHEL y cols., 2007). 
Figura 1 - Tarea de captación oculomotora

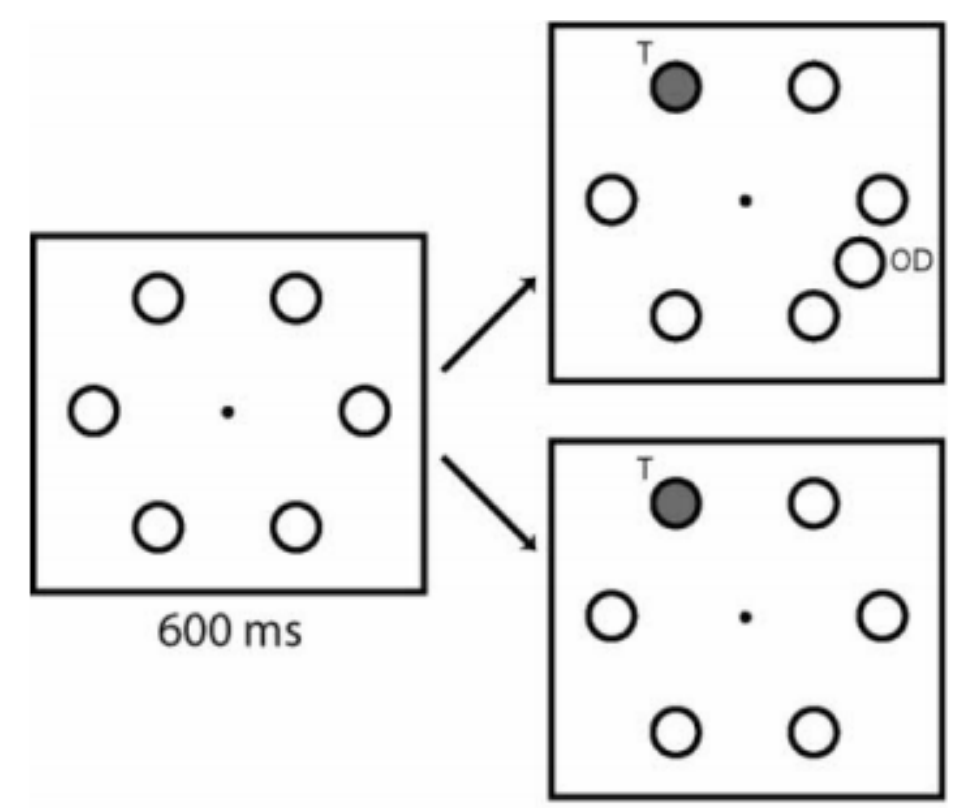

$1200 \mathrm{~ms}$

Fonte: Tomada de Van der Stigchel y cols. (2007)

- Tarea de seguimiento/persecución (pursuits): En este tipo de tarea los participantes tienen que seguir con la vista un estímulo objetivo, sin mover la cabeza. Se trata de comprobar, principalmente, si son capaces de mantener la atención sostenida.

El empleo de estas tareas ha permitido hallar resultados importantes con respecto al TDAH. Como hemos visto con anterioridad, la impulsividad es una de las características nucleares del TDAH. Si definimos esta impulsividad en términos de incapacidad para retrasar respuestas a estímulos ambientales novedosos, los movimientos oculares, o sacádicos, proporcionan una

medida ideal de las respuestas inhibitorias, ya que fallos en la inhibición están directamente reflejados en respuestas oculares erróneas (VAN DER STIGCHEL y cols., 2007).

A modo de síntesis, a continuación enumeramos los principales resultados encontrados mediante el uso de tareas de seguimiento visual (o visual tracking): en primer lugar, aunque las personas con TDAH tienen un desempeño anormal en ciertas tareas que implican inhibición de respuestas, no todos sus movimientos oculares son anormales (MUNOZ y cols., 2003; ROSS y cols., 1994; VAN DER STIGCHEL y cols., 2007); en segundo lugar, las personas con TDAH presentan dificultades a la hora de realizar fijaciones prolongadas (GOULD, BASTAIN, ISRAEL, HOMMER y CASTELLANOS, 2001; Munoz y cols., 2003); en tercer lugar, el TDAH parece estar asociado con un déficit significativo en la inhibición sacádica (FEIFEL y cols., 2004; FRIED y cols., 2014); en cuarto lugar, las investigaciones realizadas con niños con TDAH mediante la tarea antisacádica, han revelado que presentan tiempos de reacción más elevados y mayor proporción de prosacádicos erróneos que el grupo de control (VAN DER STIGCHEL y cols., 2007); y, por último, los sujetos con TDAH parecen mejorar cuando están siendo medicados con metilfenidato, aspecto éste que se refleja en sus movimientos oculares (FRIED y cols., 2014).

\subsection{Movimientos oculares y lectura en el TDAH}

No cabe duda de las implicaciones que estos resultados tienen respecto a la competencia lectora. Como hemos visto, los sujetos con TDAH presentan dificultades a la hora de realizar fijaciones por periodos prolongados de tiempo, ya que el TDAH está asociado con déficits significativos en la inhibición sacádica (FEIFEL y cols., 2004; FRIED y cols., 2014). Esto quiere decir que las personas afectadas por este trastorno presentan mayores dificultades que los no afectados para mantener la vista fija en un punto y 
evitar desviarla hacia estímulos distractores. Mostofosky y colaboradores (2001) detectaron, además, que los niños diagnosticados con TDAH a menudo miran a la localización objetivo de manera prematura, es decir, antes incluso de que aparezca la señal que marca el inicio de cada ensayo. A su vez, en este mismo estudio se descubrió que los niños con TDAH cometían de manera significativa más errores direccionales que los niños del grupo control. Sin embargo, al analizar las métricas de los sacádicos intrusivos, Munoz y colaboradores (2003) encuentran que éstas son normales, o casi normales, lo que indica que el circuito generador de los sacádicos (el cual depende del tronco cerebral) funciona correctamente en personas afectadas de TDAH. Estos mismos autores sugieren que los déficits hallados en los movimientos oculares de sujetos con TDAH son equiparables a los encontrados en pacientes con lesiones del lóbulo frontal.

Teniendo en cuenta estos resultados, las autoras de este artículo realizaron un estudio (véase ESCUDERO y MARTÍNEZ, 2016) cuyo objetivo era analizar los patrones de movimientos oculares durante la lectura de textos narrativos en niños de $5^{\circ}$ y $6^{\circ}$ de Primaria diagnosticados con TDAH, en comparación con niños sin TDAH. Como ya comentamos al comienzo de este artículo, la comprensión de la narración permite estudiar algunos de los déficits cognitivos que muestran estos sujetos, por lo que resulta de gran interés para la aplicación de esta metodología. En dicho estudio pretendíamos analizar los movimientos oculares (número de fijaciones, duración de las mismas y regresiones) durante la lectura de textos narrativos y, más específicamente, durante la lectura de las oraciones críticas (responsables de la generación de las inferencias, tanto explicativas como elaborativas). Como material se utilizaron 6 textos narrativos, de 10 oraciones cada uno, con la misma estructura causal. Cada uno de los textos tenía dos versiones: una en la que la oración crítica generaba una inferencia de naturaleza causal explicativa (necesaria para la coherencia global) y otra en la que la oración crítica generaba una inferencia de naturaleza causal predictiva. La posición de la oración crítica oscilaba entre la sexta y novena oración, según el texto. En ambos casos, y con el objetivo de dar una continuidad y resolución coherente a la historia, la inferencia (o target) era la misma (por ejemplo, manchar). A continuación se muestra un ejemplo de los textos empleados:

1. Carmen se había vestido y preparado para ir aquella noche a la fiesta.

2. Llevaba un bonito vestido de seda azul con unos pendientes a juego.

3. Se había pasado horas en el baño arreglándose para aquella noche.

4. Había estado todo el día trabajando en las obras de reforma de su casa.

5. A pesar de estar todo desbarajustado y las pareces recién pintadas, tenía ganas de salir.

6. (a) Estaba pasando por un pasillo muy estrecho cuando empezó a gritar con gran rabia. (Oración crítica explicativa) Inferencia: manchar.

(b) Iba caminando hacia la puerta, cuando pasó por un pasillo muy estrecho y angosto. (Oración crítica predictiva) Inferencia: manchar.

7. De repente, se dio cuenta de que el vestido se había manchado al arrimarse a una pared.

8. El vestido se había manchado por uno de los lados.

9. Carmen se llevó un gran disgusto.

10. A pesar de todo, se cambió de vestido y salió aquella noche.

(Adaptado de Fincher-Kiefer, 1993)

Durante la lectura de los textos se fueron registrando los movimientos oculares. Tras la lectura de cada texto, aparecía una pregunta de comprensión y, al final, se pedía a cada sujeto que hiciera un breve resumen del texto. En dicho resumen se analizó el nivel de comprensión, así como la inclusión de inferencias correctas o erróneas. Basándonos en resultados de investigaciones anteriores, según los cuales los niños con TDAH muestran dificultades importantes para la generación de inferencias causales (e.g., BERTHIAUME, LORCH y MILICH, 2010; VAN NESTE y cols., 2014), partíamos 
de la hipótesis de que estos niños mostrarían patrones diferentes a los del grupo control en dichas oraciones críticas. Los resultados mostraron que los niños diagnosticados con TDAH realizaban significativamente un menor número de fijaciones en las oraciones críticas en comparación con el grupo control y, más especialmente, en las que generaban la inferencia causal explicativa. Además, este hecho correlacionaba positivamente con una peor calidad en el resumen; es decir, un menor número de fijaciones en la oración crítica correlacionaba positivamente con una peor calidad en el resumen y/o inclusión de inferencias erróneas. Asimismo, la duración de las fijaciones, en general, fue mayor, en comparación con los niños del grupo control, como también lo fue el tiempo total de lectura. Por otra parte, otro resultado revelador fue que los niños con TDAH mostraron un mayor número de fijaciones en partes del texto que no eran relevantes para la comprensión global de la historia mostrando, por tanto, un patrón de movimientos oculares más errático. Por último, el patrón de regresiones fue también más errático en los niños con TDAH, que registraron mayor número de regresiones a partes irrelevantes del texto.

En definitiva, los resultados de este estudio están en línea con estudios previos, según los cuales los niños con TDAH presentan déficits significativos en la inhibición sacádica, así como mayor dificultad que los niños sin TDAH para mantener la vista fija en un punto, evitar desviarla hacia estímulos distractores, o cometer más errores direccionales (FEIFEL y cols., 2004; FRIED y cols., 2014; MOSTOFOSKY y cols., 2001). Además, confirman los resultados de estudios previos, según los cuales los niños con TDAH tienen dificultades para generar inferencias causales (e.g., BERTHIAUME, LORCH y MILICH, 2010; VAN NESTE y cols., 2014). Las implicaciones que estos resultados tienen a nivel educativo son muy relevantes, pues no cabe duda que la imposibilidad para realizar correctamente inferencias explicativas impide la construcción de una representación coherente de la historia. No obstante, las conclusiones de este estudio están limitadas al uso de historias breves, por lo que sería deseable seguir trabajando en esta línea, con otro tipo de textos, o textos más amplios.

Realmente son escasos los estudios sobre comprensión lectora y movimientos oculares en TDAH. Al que acabamos de presentar, debemos añadir el realizado por Deans, Laughlin, Brubaker, Gay y Krug (2010). En su trabajo, estos autores tenían como objetivo examinar la utilidad clínica de los movimientos oculares para el diagnóstico diferencial del TDAH y el trastorno de lectura (RD). Entre la muestra incluyeron a 27 niños diagnosticados con TDAH, 20 que cumplían los criterios para el diagnóstico de trastorno de lectura, y niños 30 sin diagnóstico clínico como grupo control. Las edades de los participantes oscilaban entre los 6 a 12 años. En consonancia con investigaciones anteriores, los niños del grupo RD mostraron un tiempo de lectura más lento, mayor duración de las fijaciones y más movimientos oculares atípicos, en comparación con el grupo control. Por su parte, los niños con TDAH también mostraron un patrón más atípico en comparación con el grupo control. La única diferencia significativa entre los grupos de TDAH y RD estaba en el tiempo total de lectura. Los resultados de un análisis discriminante revelaron que a menos del $60 \%$ de los participantes se les dio una correcta clasificación diagnóstica basada en el tiempo de lectura total y en la proporción de movimientos sacádicos. Por lo tanto, estos autores concluyen que la metodología de movimientos oculares tiene limitaciones importantes, al menos, como criterio diagnóstico del TDAH en comparación con el trastorno de lectura.

Pese a que estos últimos resultados prestan un apoyo limitado al uso de esta metodología en niños con TDAH, pensamos que se hace necesaria más investigación al respecto. Son muchas las variables que pueden afectar a la obtención de resultados concluyentes, que pasan por las propias condiciones de la aplicación de la tarea (e.g., la motivación de los niños durante la misma, 
el manejo de las instrucciones que se proporcionan...), los estímulos, el tipo de variables que se utilizan para los análisis, o los análisis mismos. Si bien hemos descrito brevemente algunas de las variables más básicas, existen multitud de variables que pueden emplearse en movimientos oculares dependiendo del objetivo de estudio (véase, para una revisión más extensa, HOLMQVIST y NYSTRÖM, 2011). Por ello, la investigación futura debería ir encaminada a analizar todas estas cuestiones, así como poder ayudar a solventar las dificultades que puedan encontrarse por el camino. Se trata, en definitiva, de trabajar para que los beneficios asociados al uso de esta metodología excedan los costes de la misma.

\section{Conclusiones}

Este trabajo tenía como principal objetivo mostrar la utilidad de la metodología de los movimientos oculares para el estudio del TDAH y, más concretamente, para analizar las dificultades de lectura en estos niños. Como mencionamos al comienzo de este artículo, la comprensión narrativa es una forma de poder aproximarse al estudio de los procesos de orden superior $y$, por tanto, a los déficit que presentan estos niños. Son diversos los estudios que han mostrado que estos niños presentan dificultades importantes en competencia lectora como, por ejemplo, poder juzgar el contenido de un texto, sintetizar, resumir, esquematizar, realizar inferencias, comprender las conexiones causales o las metas de los personajes, así como distinguir la información relevante (LORCH y cols., 2007; LORCH, DIENER, SÁNCHEZ, MILICH, WELSH y VAN DEL BROEK, 1999; MIRANDA y cols., 2005; RENZ, LORCH, MILICH, LEMBERGER, BODNER y WELSH, 2003; VAN NESTE y cols., 2015). Por ello, y dado que competencia lectora y rendimiento académico van íntimamente relacionados, no cabe duda de que todo trabajo en esta línea es insuficiente.
Por otra parte, hemos visto que los movimientos oculares son un indicador observable y cuantificable de los procesos cognitivos subyacentes, supuestamente alterados en el TDAH. Hemos revisado brevemente los resultados de varios estudios que apuntan en esta dirección y hemos visto cómo esta metodología ha ido ganando un espacio importante dentro del estudio de la lectura. Sin embargo, su aplicación para el estudio de las dificultades de lectura en el TDAH es aún muy limitado. Creemos que es importante seguir trabajando en esta línea, pues no cada duda de que su manejo permitía, no solo conocer más en profundidad las dificultades que tienen estos niños, sino también poder realizar las adaptaciones necesarias en los textos o materiales, probar su validez, o trabajar en el desarrollo de habilidades visuales, a través, por ejemplo del uso de terapias visuales. Esto implica seguir investigando en todos los aspectos que afectan al uso de esta metodología, ya sea su aplicación (a través del uso de instrucciones adaptadas para estos niños), el tipo de estímulo o material utilizado, el tipo de variable/s a analizar o los análisis estadísticos utilizados.

\section{Referencias}

AMERICAN PSYCHIATRIC ASSOCIATION. Guía de consulta de los criterios diagnósticos del DSM-5. Madrid: Médica Panamericana, 2013.

ARMSTRONG, I.; MUNOZ, D. P. Attentional blink in adults with attention-deficit hyperactivity disorder. Influence of eye movements. Experimental Brain Research, v. 152, n. 2, p. 243-250, 2003.

ATIENZA, J. D. Comorbilidad en el TDAH. Revista De Psiquiatría y Psicología del Niño y del Adolescente, v. 6, n. 1, p. 44-55, 2006.

BARKLEY, R. A. Attention-deficit hyperactivity disorder. A handbook for diagnosis and treatment. 3rd ed. New York: Guilford Press, 2006.

BERTHIAUME, K.; LORCH, E.; MILICH, R. Getting clued in: inferential processing and comprehension monitoring in boys with ADHD. Journal of Attention Disorders, v. 14, n. 1, p. 31-42, 2010. 
BLYTHE, H.; JOSEPH, S. Children's eye movements during reading. In: LIVERSEDGE, S. P.; GILCHRIST, I. D.; EVERLING, S. (Eds.). Oxford handbook on eye movements. Oxford, England: Oxford University Press, 2011. p. 643-662.

BUCCI, M.P.; SEASSAU, M.; LARGER, S.; BUI-QUOC, E.; GERARD, C. L. Effect of visual attention on postural control in children with attention-deficit/hyperactivity disorder. Research in Developmental Disabilities, v, 35, p. 1292-1300, 2014.

BYLSMA, F. W.; PIVIK, R. T. The effects of background illumination and stimulant medication on smooth pursuit eye movements of hyperactive children. Journal of Abnormal Child Psychology, v. 17, n. 1, p. 73-90, 1989.

DEANS, P.; O’LAUGHLIN, L.; BRUBAKER, B.; GAY, N.; KRUG, D. Use of Eye Movement Tracking in the Differential Diagnosis of Attention Deficit Hyperactivity Disorder (ADHD) and Reading Disability. Psychology, v. 1, p. 238-246, 2010.

ESCUDERO, I.; LEÓN, J. A.; MORERA, Y. Are discourse comprehension and cognitive processes influenced by the type of language language (English and Spanish)? A crosslanguage study based on elaborative inferences generation. Revista de Investigación Educativa, v. 31, n. 1, p. 223-237, 2013.

ESCUDERO, I.; MARTÍNEZ, P. Eye movement patterns during inference generation in narrative texts among children with ADHD. Comunicación enviada al 57th Annual Meeting of The Psychonomic Society. 17-20 de noviembre 2016, Boston, M. A.

FEIFEL, D.; FARBER, R. H.; CLEMENTZ, B. A.; PERRY, W.; ANLLO-VENTO, L. Inhibitory deficits in ocular motor behavior in adults with attention deficit/hyperactivity disorder. Biological Psychiatry, v. 56, n. 5, p. 333-339, 2004

FINCHER-KIEFER, R. The role of predictive inferences in situation model construction. Discourse Processes, v. 16, p. 99-124, 1993.

FRIED, M.; TSITSIASHVILI, E.; BONNEH, Y. S.; STERKIN, A.; WYGNANSKI-JAFFE, T.; EPSTEIN, T.; COLS. ADHD subjects fail to suppress eye blinks and microsaccades while anticipating visual stimuli but recover with medication. Vision Research, v. 101, p. 62-72, 2014.

GOULD, T.D.; BASTAIN, T.M.; ISRAEL, M.E.; HOMMER, D.W.; CASTELLANOS, F.X. Altered performance on an ocular fixation task in attention deficit/hyperactivity disorder. Biological Psychiatry, v. 50, n. 8, p. 633-635, 2001.

HANISCH, C.; RADACH, R.; HOLTKAMP, K.; HERPERTZ-DAHLMANN, B.; KONRAD, K. Oculomotor inhibition in children with and without attention-deficit hyperactivity disorder (ADHD). J Neural Transm (Vienna), v. 113, n. 5, p. 671-684, 2006.
HOLMQVIST, K.; NYSTRÖM, M. Eye tracking. A Comprehensive Guide to Methods and Measures. New York: Oxford University Press, 2011.

KENDEOU, P.; BOHN-GETTLER, C.; WHITE, M.; VAN DEN BROEK, P. Children's inference generation across different media. Journal of Research in Reading, v. 31, n. 3, p. 259-272, 2008.

KLEIN, C.; RASCHKE, A.; BRANDENBUSCH, A. Development of pro- and antisaccades in children with attention-deficit hyperactivity disorder (ADHD) and healthy controls. Psychophysiology, v. 40, n. 1, p. 17-28, 2003.

LEÓN, J. A.; ESCUDERO, I. Understanding Causality in Science Discourse for Middle and High School Students. Summary Task as a Strategy for Improving Comprehension. In: SANTI, K. L.; REED, D. K. (Eds.). Improving Reading Comprehension of Middle and High School Students. Switzerland: Springer International Publishing, 2015. p. 75-98.

LORCH, E.; BERTHIAUME, K.; MILICH, R.; VAN DEN BROEK, P. Story comprehension impairments in children with Attention-Deficit/Hyperactivity Disorder. Children's comprehension problems in oral and written language: A cognitive perspective. New York: Guilford Press, 2007. p. 128-156.

LORCH, E.; DIENER, M.; SANCHEZ, R.; MILICH, R.; WELSH, R.; VAN DEN BROEK, P. The effects of story structure on the recall of stories in children with attention deficit hyperactivity disorder. Journal of Educational Psychology, v. 91, n. 2, p. 273-283, 1999.

MAYES, S. D.; CALHOUN, S. L.; CROWELL, E. W. Learning disabilities and ADHD: Overlapping spectrum disorders. Journal of Learning Disabilities, v. 33, n. 5, p. 417-424, 2000. MCCONKIE, G. W.; ZOLA, D.; GRIMES, J.; KERR, P. W.; BRYANT, N. R.; WOLFF, P. M. Children's eye movements during reading. In: Stein, J. F. (Ed.). Vision and visual dyslexia. Boston: CRC Press, 1991. p. 251-262.

MIRANDA, A.; FERNÁNDEZ, M. I.; GARCÍA, R.; ROSELLÓ, B.; COLOMER, C. Habilidades lingüísticas y ejecutivas en el Trastorno por Déficit de Atención (TDAH) y en las Dificultades de Comprensión Lectora (DCL). Psicothema, v. 23, n. 4, p. 688-694, 2011.

MIRANDA, A.; GARCÍA, R.; SORIANO, M. Habilidad Narrativa de los niños con Trastorno por Déficit de Atención con Hiperactividad. Psicothema, v. 17, n. 2, p. 227-232, 2005.

MOSTOFSKY, S. H.; LASKER, A. G.; CUTTING, L. E. Oculomotor Abnormalities in Attention Deficit Hyperactivity Disorder: A Preliminary Study. Neurology, v. 57, n. 3, p. 423-430, 2001. MUNOZ, D. P.; ARMSTRONG, I. T.; HAMPTON, K. A.; MOORE, K. D. Altered control of visual fixation and saccadic eye movements in attention-deficit hyperactivity disorder. Journal of Neurophysiology, v. 90, n. 1, p. 503-514, 2003. 
ORJALES, I. Déficit de Atención con Hiperactividad. Manual para Padres y Educadores. Madrid: CEPE, 2011

RAYNER, K. Eye movements and the perceptual span in beginning and skilled readers. Journal of Experimental Child Psychology, v. 41, p. 211-236, 1986.

RAYNER, K. Eye Movements in Reading and Information Processing: 20 Years of Research. Psychological Bulletin, v. 124, n. 3, p. 372-422, 1998.

RAYNER, K. Eye movements in reading: Models and data. Journal of Eye Movement Research, v, 2, n. 5, p. 1-10, 2009.

RENZ K.; LORCH, E. P.; MILICH, R.; LEMBERGER, C.; BODNER, A.; WELSH, R. On-line story representation in boys with attention deficit hyperactivity disorder. Journal of Abnorm Child Psychology, v. 31, n. 1, p. 93-104, 2003.

ROMMELSE, N. N.; VAN DER STIGCHEL, S.; SERGEANT, J. A. A review on eye movement studies in childhood and adolescent psychiatry. Brain and Cognition, v. 68, p. 391-414, 2008.

ROSS, R. G.; HOMMER, D. W.; BREIGER, D.; VARLEY, C.; RADANT, A. Eye movement task related to frontal lobe functioning in children with attention deficit disorder. Journal of the American Academy of Child \& Adolescent Psychiatry, v. 33, n. 6, p. 869-874, 1994.

ROTHLIND, J. C.; POSNER, M. I.; SCHAUGHENCY, E. A. Lateralized control of eye movements in attention deficit hyperactivity disorder. Journal of Cognitive Neuroscience, v. 3, n. 4, p. 377-381, 1991.

TAYLOR, S. E. Eye movements while reading: Facts and fallacies. American Educational Research Journal, v. 2, p. 187-202, 1965.

VAN DER STIGCHEL, S.; ROMMELSE, N. N.; DEIJEN, J. B.; GELDOF, C. J.; WITLOX, J.; OOSTERLAAN, J.; SERGEANT, J. A.; THEEUWES, J. Oculomotor capture in ADHD. Cognitive Neuropsychology, v. 24, n. 5, p. 535-549, 2007.

VAN NESTE, J.; HAYDEN, A.; LORCH, E. P.; MILICH, R. Inference generation and story comprehension among children with ADHD. Journal of abnormal child psychology, v. 43 , n. 2, p. 259-270, 2015.

VAQUERIZO-MADRID, J.; ESTÉVEZ-DÍAZ, F.; POZO-GARCÍA, A. El lenguaje en el trastorno por déficit de atención con hiperactividad: competencias narrativas. Revista Neurología, v. 41, n. 1, p. $83-89,2005$.
WILLCUTT, E. G.; PENNINGTON, B. F.; OLSON, R. K.; DEFRIES, J. C. Understanding comorbidity: A twin study of reading disability and attention-defi cit/hyperactivity disorder. American Journal of Medical Genetics (Neuropsychiatric Genetics), v. 8, p. 709-714, 2007.

Recebido em 25/04/2016

Aceito em $31 / 05 / 2016$. 